\title{
Modelling and solutions to the linear stability of a detonation wave in the kinetic frame
}

\author{
M. Pandolfi Bianchi \\ Dipartimento di Matematica, Politecnico di Torino, C. Duca degli Abruzzi 24, 10129 Torino, Italy \\ A. J. Soares \\ Departamento de Matemática, Universidade do Minho Gualtar, 4710-057 Braga, Portugal
}

\begin{abstract}
The analysis of linear stability of a steady detonation wave is formulated for the first time at the kinetic level in the frame of the Boltzmann equation extended to reacting gases. Within this context and for a reversible reaction, the stability problem is carried out, in agreement with most classical papers on gas detonation, through a normal mode approach for the onedimensional disturbances of the steady wave solution, and an acoustic radiation condition at the final equilibrium as closure condition. The proposed modelling leads to an initial value problem, constituted by the linearized reactive Euler equations in the perturbed shock frame with related Rankine-Hugoniot conditions, which can be solved by means of a proper numerical technique. An application is provided for an elementary bimolecular reaction.
\end{abstract}

AMS codes: 35B35; 76P05; 80A32; 82C40; 76P05.

Keywords: Boltzmann equation. Chemical reactions. Detonation solution. Linear stability.

\section{Introduction}

The differential system of reactive stability equations and Rankine-Hugoniot conditions for a steady detonation wave arising in a reacting gas is deduced for the first time within the kinetic frame of the Boltzmann equation, and is solved for an elementary explosive reaction of the Hydrogen-Oxygen chain, assuming an acoustic radiation constraint as closure condition [1]. The proposed mathematical formulation is justified on the one hand by the knowledge of a kinetic model of the steady wave [2], and on the other by the possibility of recovering a methodology widely discussed in the hydrodynamical approaches to the detonation linear stability problem $[1,3]$. With respect to classical studies on detonation stability which mainly consider ideal gases with one-step irreversible reaction governed by an Arrhenius kinetics to model the combustion process, the kinetic device has the advantage of working with a more detailed chemistry mechanism, providing also a mesoscopic picture of the rather complex phenomenology.

In Sections 2, 3 and 4 the relevant mathematical and chemical features of the kinetic model related to the gas description [4], to the governing equations and to the detonation wave structure $[2,5]$, respectively, are reviewed and interpreted in order to justify the formulation of the stability problem proposed in the modelling of Section 5. 
The actual solution to the initial value problem consisting of the stability equations and related Rankine-Hugoniot conditions, together with a pertinent closure condition is solved for the application dealt with in Section 6.

\section{Kinetic and chemical preliminaries}

A mixture of constituents $A_{i}, i=1, \ldots, 4$, undergoing the chemical reaction $A_{1}+A_{2} \rightleftharpoons A_{3}+A_{4}$, with molecular masses such that $m_{1}+m_{2}=m_{3}+m_{4}$ and binding energy difference $E=$ $E_{3}+E_{4}-E_{1}-E_{2}>0$ is described by means of the kinetic model of paper [4] which is here revisited in view of the formulation of the stability problem of Section 5 .

Reactive Boltzmann equation. The microscopic state of the mixture is defined by the one-particle distribution function $f_{i}(t, \boldsymbol{x}, \boldsymbol{v}), t \in \mathbb{R}_{+}, \boldsymbol{x} \in \mathbb{R}^{3}, \boldsymbol{v} \in \mathbb{R}^{3}$, for constituent $A_{i}$, which satisfies the extended Boltzmann equation

$$
\frac{\partial f_{i}}{\partial t}+\boldsymbol{v} \cdot \nabla f_{i}=\mathcal{Q}_{i}[\boldsymbol{f}]+\mathcal{R}_{i}[\boldsymbol{f}], \quad i=1, \ldots, 4,
$$

where $\boldsymbol{f}=\left(f_{1}, f_{2}, f_{3}, f_{4}\right)$ and $\mathcal{Q}_{i}[\boldsymbol{f}], \mathcal{R}_{i}[\boldsymbol{f}]$ are the collision terms referred to elastic scattering and inelastic collisions with chemical reaction, respectively, whose form is detailed only for some of them, $\mathcal{R}_{2}[\boldsymbol{f}]$ being analogous to $\mathcal{R}_{1}[\boldsymbol{f}]$, and $\mathcal{R}_{4}[\boldsymbol{f}]$ to $\mathcal{R}_{3}[\boldsymbol{f}]$,

$$
\begin{aligned}
& \mathcal{Q}_{i}[\boldsymbol{f}]=\sum_{j=1}^{4} \int_{\mathbb{R}^{3}} \int_{\mathcal{S}} V I_{i j}^{i j} f_{i}\left(\boldsymbol{v}_{i j}\right) f_{j}\left(\boldsymbol{w}_{i j}\right) d \Omega^{\prime} d \boldsymbol{w}-f_{i}(\boldsymbol{v}) \int_{\mathbb{R}^{3}} \int_{\mathcal{S}} V I_{i j}^{i j} f_{j}(\boldsymbol{w}) d \Omega^{\prime} d \boldsymbol{w}, \\
& \mathcal{R}_{1}[\boldsymbol{f}]=\left(\frac{\mu_{12}}{\mu_{34}}\right)^{3} \int_{\mathbb{R}^{3}} \int_{\mathcal{S}} V I_{12}^{34} f_{3}\left(\boldsymbol{v}_{1}\right) f_{4}\left(\boldsymbol{w}_{1}\right) d \Omega^{\prime} d \boldsymbol{w}-f_{1}(\boldsymbol{v}) \int_{\mathbb{R}^{3}} \int_{\mathcal{S}} V I_{12}^{34} f_{2}(\boldsymbol{w}) d \Omega^{\prime} d \boldsymbol{w}, \\
& \mathcal{R}_{3}[\boldsymbol{f}]=\left(\frac{\mu_{34}}{\mu_{12}}\right)^{3} \int_{\mathbb{R}^{3}} \int_{\mathcal{S}} V I_{34}^{12} f_{1}\left(\boldsymbol{v}_{3}\right) f_{2}\left(\boldsymbol{w}_{3}\right) d \Omega^{\prime} d \boldsymbol{w}-f_{3}(\boldsymbol{v}) \int_{\mathbb{R}^{3}} \int_{\mathcal{S}} V I_{34}^{12} f_{4}(\boldsymbol{w}) d \Omega^{\prime} d \boldsymbol{w},
\end{aligned}
$$

with $S$ unit sphere in $\mathbb{R}^{3}, I_{i j}^{i j}$ symmetric differential scattering cross sections, $I_{12}^{34}, I_{34}^{12}$ reactive cross sections for the forward and backward reaction, depending on relative pre and post collision velocities $V$ and $V^{\prime}$ with unit vectors $\Omega$ and $\Omega^{\prime} ; \mu_{i j}=m_{i} m_{j} /\left(m_{1}+m_{2}\right) ; \boldsymbol{v}_{i j}, \boldsymbol{w}_{i j}$ and $\boldsymbol{v}, \boldsymbol{w}$ postcollision velocities of elastic and reactive interactions, depending on the incoming velocities.

Properties of the collision terms and equilibrium conditions. The mathematical and chemical aspects of the collision terms and equilibrium conditions are here focused in Properties 1-8. The proof of Properties 1-7 is omitted (see Ref. [4]).

Property 1 The elastic collision terms are such that $\int_{\mathbb{R}^{3}} \mathcal{Q}_{i}[\boldsymbol{f}] d \boldsymbol{v}=0$.

Conservation of constituent number density $n_{i}=\int_{\mathbb{R}^{3}} f_{i} d \boldsymbol{v}$ during elastic collisions is thus assured.

Property 2 For the collision invariants $\psi_{i}(\boldsymbol{v})=m_{i}, m_{i} \boldsymbol{v}, E_{i}+m_{i} \boldsymbol{v}^{2} / 2$, it holds

$$
\sum_{i=1}^{4} \int_{\mathbb{R}^{3}} \psi_{i}(\boldsymbol{v})\left(\mathcal{Q}_{i}[\boldsymbol{f}]+\mathcal{R}_{i}[\boldsymbol{f}]\right)(\boldsymbol{v}) d \boldsymbol{v}=0 .
$$


Collision terms are then consistent with conservation of mass, momentum and total energy.

Property 3 The reactive collision terms fulfill the following equalities

$$
\int_{\mathbb{R}^{3}} \mathcal{R}_{1}[\boldsymbol{f}](\boldsymbol{v}) d \boldsymbol{v}=\int_{\mathbb{R}^{3}} \mathcal{R}_{2}[\boldsymbol{f}](\boldsymbol{v}) d \boldsymbol{v}=-\int_{\mathbb{R}^{3}} \mathcal{R}_{3}[\boldsymbol{f}](\boldsymbol{v}) d \boldsymbol{v}=-\int_{\mathbb{R}^{3}} \mathcal{R}_{4}[\boldsymbol{f}](\boldsymbol{v}) d \boldsymbol{v} .
$$

Therefore the reactive terms link the consumption rate of reactants to the formation rate of products, and, at the same time, assure the correct chemical exchanges predicted by the reaction.

Property 4 For the chemical exchange rates $\tau_{i}$ defined by $\tau_{i}=-\nu_{i} \int_{\mathbb{R}^{3}} \mathcal{R}_{1}[\boldsymbol{f}](\boldsymbol{v}) d \boldsymbol{v}$, with stoichiometric coefficients $\nu_{1}=\nu_{2}=-\nu_{3}=-\nu_{4}=-1$, it holds $\tau_{1}=\tau_{2}=-\tau_{3}=-\tau_{4}$.

The correct link among the exchange rates of both reactants and products is thus assured.

Property 5 The rate $\tau_{1}$ splits in $\tau_{1}=\tau_{f}-\tau_{b}$, with forward and backward rates given by

$$
\begin{aligned}
\tau_{f} & =\left(\frac{\mu_{12}}{\mu_{34}}\right)^{3} \int_{\mathbb{R}^{3}} \int_{\mathbb{R}^{3}} \int_{\mathcal{S}} V I_{34}^{12}\left(V, \Omega, \Omega^{\prime}\right) f_{3}\left(\boldsymbol{v}_{1}\right) f_{4}\left(\boldsymbol{w}_{1}\right) d \Omega^{\prime} d \boldsymbol{w} d \boldsymbol{v}, \\
\tau_{b} & =\int_{\mathbb{R}^{3}} \int_{\mathbb{R}^{3}} \int_{\mathcal{S}} V I_{34}^{12}\left(V, \Omega, \Omega^{\prime}\right) f_{1}(\boldsymbol{v}) f_{2}(\boldsymbol{w}) d \Omega^{\prime} d \boldsymbol{w} d \boldsymbol{v} .
\end{aligned}
$$

The form of $\tau_{1}$ due to Eq. (2b) shows the detailed balance of source and sink terms of the chemical interactions, coherently with its classical definition (see Ref. [6]).

Property 6 The equilibrium condition $\mathcal{Q}_{i}[\boldsymbol{f}]+\mathcal{R}_{i}[\boldsymbol{f}]=0$, for $i=1, \ldots, 4$, is equivalent to the the vanishing of both elastic and reactive collision terms, namely $\mathcal{Q}_{i}[\boldsymbol{f}]=0$ and $\mathcal{R}_{i}[\boldsymbol{f}]=0$.

The vanishing of $\mathcal{Q}_{i}[\boldsymbol{f}]$ means that elastic collisions proceed without modifying the distribution $f_{i}$, thus a state of mechanical equilibrium is reached. The vanishing of $\mathcal{R}_{i}[\boldsymbol{f}]$ means that reacting interactions occur without varying $f_{i}$, as well, and thus a state of chemical equilibrium is defined.

Property 7 The mechanical equilibrium is assured by the Maxwellian distributions

$$
\widetilde{f}_{i}(\boldsymbol{v})=n_{i}\left(\frac{m_{i}}{2 \pi k_{B} T}\right)^{3 / 2} \exp \left(-\frac{m_{i}(\boldsymbol{v}-\boldsymbol{u})^{2}}{2 k_{B} T}\right)
$$

with uncorrelated $n_{i}$. The chemical equilibrium is characterized by Maxwellians (6) constrained by $\left(m_{3} m_{4}\right)^{3} \widetilde{f}_{1}(\boldsymbol{v}) \widetilde{f}_{2}(\boldsymbol{w})=\left(m_{1} m_{2}\right)^{3} \widetilde{f}_{3}\left(\boldsymbol{v}_{1}\right) \widetilde{f}_{4}\left(\boldsymbol{w}_{1}\right)$, which is equivalently to the mass action law

$$
\frac{n_{1}^{\mathrm{eq}} n_{2}^{\mathrm{eq}}}{n_{3}^{\mathrm{eq}} n_{4}^{\mathrm{eq}}}=\left(\frac{m_{1} m_{2}}{m_{3} m_{4}}\right)^{3 / 2} \exp \left(\frac{E}{k_{B} T}\right)
$$


Above, $k_{B}$ is the Boltzmann constant, $n_{i}^{\text {eq }}$ the equilibrium number density, $\boldsymbol{u}$ and $T$ the mean velocity and temperature. Equation (7) reproduces the mass action law of chemical kinetics.

An equilibrium description more pertinent to the chemical kinetics is based on the affinity [6] which gives a measure of the distance of the gas system from the chemical equilibrium, that is

$$
\mathcal{A}=-\sum_{i=1}^{4} \nu_{i} \mu_{i}, \quad \mu_{i}=E_{i}-k_{B} T\left[\frac{3}{2} \ln T-\ln n_{i}+\frac{3}{2} \ln \left(\frac{2 \pi m_{i} k_{B}}{h^{2}}\right)\right],
$$

$h$ being the Planck constant, and $\mu_{i}$ the chemical interaction potential when the gas molecules are endowed with translational degrees of freedom only. If $\mathcal{A}=0$, the chemical equilibrium reads

$$
\mu_{1}^{\mathrm{eq}}+\mu_{2}^{\mathrm{eq}}=\mu_{3}^{\mathrm{eq}}+\mu_{4}^{\mathrm{eq}} .
$$

Property 8 The kinetic form of the affinity is $\mathcal{A}=k_{B} T \ln \left(\frac{n_{1} n_{2} n_{3}^{\mathrm{eq}} n_{4}^{\mathrm{eq}}}{n_{3} n_{4} n_{1}^{\mathrm{eq}} n_{2}^{\mathrm{eq}}}\right)$ and the chemical equilibrium condition $\mathcal{A}=0$ is equivalent to the mass action law (7).

Proof. Note first that the kinetic form of $\mathcal{A}$ is obtained from the stoichiometric definition $(8)_{1}$ with chemical potentials $(8)_{2}$, and from Eq. (7) for the mass ratio. In the second statement, the necessary condition follows from the stoichiometric and kinetic definitions of $\mathcal{A}$ inserting Eqs. $(8)_{2}$ and (9). The sufficiency is proved casting Eqs. (8) 2 and (7) into Eq. (8) $)_{1}$.

Reaction rate. The gas chemical composition is specified by the progress variable of the model, say $n_{1}$, since $A_{1}$ is a product of the exothermic reaction, increasing from 0 when $A_{1}$ is absent, to the equilibrium value $n_{1}^{\mathrm{eq}}$, according to the irreversible entropy behaviour. The rate equation of the model, obtained integrating the kinetic equation (1) over the velocity $\boldsymbol{v}$, gives the evolution of $n_{1}, d n_{1} / d t(\boldsymbol{x}, t)=\tau_{1}(\boldsymbol{x}, t)$ where $d / d t$ is the Lagrangian derivative and $\tau_{1}$, defined in Property 5 , is the reaction rate. When $\tau_{1}=0$, the concentration of each constituent is then constant due to Property 3. In a flow regime close to chemical equilibrium the distributions are assumed Maxwellian given by Eqs. (6). Adopting the reactive cross sections of paper [7], inserting the Maxwellians (6) into Eqs. (5a-b) and performing the integrations, the reation rate $\tau_{1}$ becomes

$$
\widetilde{\tau}_{1}=\left[-\varrho_{1} \varrho_{2}\left(\frac{m_{3} m_{4}}{m_{1} m_{2}}\right)^{5 / 2} \exp \left(-\frac{E}{k_{B} T}\right)+\varrho_{3} \varrho_{4}\right] \widehat{S},
$$

where $\widehat{S}$ is a weight function depending on temperature and threshold velocity (see Ref. [5]).

\section{Macroscopic governing equations}

In this section the reactive Euler equations are deduced in the form suitable for the stability analysis of Section 5 and, due to Proposition 1, in the more conservative form convenient to treat the velocity problem of the steady detonation wave of Section 4 .

Macroscopic observables. The macroscopic observables are defined in terms of the distribution functions by the equalities 


$$
\begin{gathered}
\varrho_{i}=\int m_{i} f_{i} d \boldsymbol{v}, \varrho_{i} u_{k}^{i}=\int m_{i} v_{k} f_{i} d \boldsymbol{v}, \quad \text { with } \quad \varrho=\sum_{i=1}^{4} \varrho_{i}, n=\sum_{i=1}^{4} n_{i}, \varrho u_{k}=\sum_{i=1}^{4} \varrho_{i} u_{k}^{i}, \\
P_{k l}^{i}=\int m_{i}\left(v_{k}-u_{k}\right)\left(v_{l}-u_{l}\right) f_{i} d \boldsymbol{v}, \quad \text { with } \quad P_{k l}=\sum_{i=1}^{4} P_{k l}^{i}, \\
p_{i}=\frac{1}{3} \int m_{i}(\boldsymbol{v}-\boldsymbol{u})^{2} f_{i} d \boldsymbol{v}, T_{i}=\frac{p_{i}}{n_{i} k_{B}}, \quad \text { with } \quad p=\sum_{i=1}^{4} p_{i}, T=\sum_{i=1}^{4} \frac{n_{i}}{n} T_{i}=\frac{p}{n k_{B}}, \\
q_{k}^{i}=\int \frac{1}{2} m_{i}(\boldsymbol{v}-\boldsymbol{u})^{2}\left(v_{k}-u_{k}\right) f_{i} d \boldsymbol{v}, \quad \text { with } \quad q_{k}=\sum_{i=1}^{4}\left[q_{k}^{i}+E_{i} n_{i}\left(u_{k}^{i}-u_{k}\right)\right] .
\end{gathered}
$$

The mixture observables, $\varrho, n, p, T$ and $u_{k}, P_{k l}, q_{k}$, are the mass density, total number density, pressure, temperature and components of mean velocity, pressure tensor, heat flux. The corresponding quantities of each constituent are denoted by the same symbols with index $i$. The term $E_{i} n_{i}\left(u_{k}^{i}-u_{k}\right)$ in expression $(11 d)_{2}$ refers to the formation energy transfer due to diffusion.

Balance equations. The macroscopic equations for constituent mass densities, momentum and total energy of the whole mixture are obtained from the Boltzmann equation (1) in the form

$$
\begin{aligned}
& \frac{\partial \varrho_{i}}{\partial t}+\nabla\left(\varrho_{i} \boldsymbol{u}_{i}\right)=m_{i} \tau_{i}, \quad i=1, \ldots, 4 \\
& \frac{\partial}{\partial t}(\varrho \boldsymbol{u})+\nabla(\varrho \boldsymbol{u} \otimes \boldsymbol{u}+\mathbb{P})=0 \\
& \frac{\partial}{\partial t}\left(\frac{3}{2} n k_{B} T+\sum_{i=1}^{4} n_{i} E_{i}+\frac{1}{2} \varrho \boldsymbol{u}^{2}\right)+\nabla\left[\boldsymbol{q}+\mathbb{P} \boldsymbol{u}+\left(\frac{3}{2} n k_{B} T+\sum_{i=1}^{4} n_{i} E_{i}+\frac{1}{2} \varrho \boldsymbol{u}^{2}\right) \boldsymbol{u}\right]=0
\end{aligned}
$$

where $\mathbb{P}$ is the mixture pressure tensor with components $P_{k l}$ and $\tau_{i}$ is the reaction rate.

Proposition 1. The four evolution equations (12a) are equivalent to the set

$$
\begin{aligned}
& \frac{\partial \varrho_{1}}{\partial t}+\nabla\left(\varrho_{1} \boldsymbol{u}_{1}\right)=m_{1} \tau_{1}, \\
& \frac{\partial}{\partial t}\left(n_{1}+n_{3}\right)+\nabla\left(n_{1} \boldsymbol{u}_{1}+n_{3} \boldsymbol{u}_{3}\right)=0, \\
& \frac{\partial}{\partial t}\left(n_{1}+n_{4}\right)+\nabla\left(n_{1} \boldsymbol{u}_{1}+n_{4} \boldsymbol{u}_{4}\right)=0, \\
& \frac{\partial}{\partial t}\left(n_{2}+n_{3}\right)+\nabla\left(n_{2} \boldsymbol{u}_{2}+n_{3} \boldsymbol{u}_{3}\right)=0 .
\end{aligned}
$$

Proof. To deduce Eqs. $(13 a-d)$ it is sufficient to divide Eqs. (12a) by $m_{i}$ and sum them separately for $i=1,3$, for $i=1,4$, and for $i=2,3$, respectively, taking into account Property 4 . Conversely, linear combinations of Eqs. $(13 a-d)$ allow to deduce the evolution equations $(12 a)$. 
Reactive Euler equations. Referring the set $(12 a-c)$ to the Maxwellians (6) and performing some algebraic manipulations, the one-space dimensional reactive Euler (RE) equations become

$$
\begin{aligned}
& \frac{\partial \varrho_{i}}{\partial t}+u \frac{\partial \varrho_{i}}{\partial x}+\varrho_{i} \frac{\partial u}{\partial x}=-m_{i} \nu_{i} \widetilde{\tau}_{1}, \quad i=1, \ldots, 4, \\
& \frac{\partial u}{\partial t}+u \frac{\partial u}{\partial x}+\frac{1}{\varrho} \frac{\partial p}{\partial x}=0, \quad \frac{\partial p}{\partial t}+u \frac{\partial p}{\partial x}+\frac{5}{3} p \frac{\partial u}{\partial x}=\eta,
\end{aligned}
$$

where $\eta=2 \sum_{i=1}^{4} \nu_{i} E_{i} \widetilde{\tau}_{1} / 3=2 E \widetilde{\tau}_{1} / 3$. Their matrix form, which will be useful in Section 5 , is

$$
\frac{\partial \boldsymbol{z}}{\partial t}+A(\boldsymbol{z}) \frac{\partial \boldsymbol{z}}{\partial x}=\boldsymbol{c}(\boldsymbol{z})
$$

with $\boldsymbol{z}=\left[\varrho_{1} \varrho_{2} \varrho_{3} \varrho_{4} u p\right]^{\mathrm{T}}$ the unknown state vector and non-zero elements of $A$ and $\boldsymbol{c}$ given by

$$
A_{i i}=u, A_{i 5}=\varrho_{i}, i=1, \ldots, 4, A_{56}=1 / \varrho, A_{65}=5 p / 3, \quad c_{i}=-\nu_{i} m_{i} \widetilde{\tau}_{1}, i=1, \ldots, 4, \quad c_{6}=\eta \text {. }
$$

Remark 1 A more conservative form of Eqs. (14a-b), which will be adopted in Section 4, can be written substituting Eqs. (14a) by Eqs. $(13 a-d)$ in one dimension and Eq. $(14 b)_{2}$ by

$$
\frac{\partial}{\partial t}\left(\frac{3}{2} n k T+\sum_{i=1}^{4} n_{i} E_{i}+\frac{1}{2} \varrho u^{2}\right)+\frac{\partial}{\partial x}\left[p u+\left(\frac{3}{2} n k_{B} T+\sum_{i=1}^{4} n_{i} E_{i}+\frac{1}{2} \varrho u^{2}\right) u\right]=0 .
$$

\section{Steady detonation waves}

The one-dimensional steady detonation with chemical reaction of reversible bimolecular type can be well described also within the kinetic frame, resorting to the so called ZND model, due to Zeldovich, von Neumann and Doering [9] extended to reversible reactions. The structure of the ZND detonation wave is represented in Fig. 1. The lead element is a non-reactive shock wave propagating with constant velocity $\mathcal{D}$, from the left to the right towards an unreacted quiescent gas mixture followed by a finite reaction zone connecting the von Newmann state $N$, where the exothermic reaction is triggered, to the final equilibrium state $S$. Inside this zone, the explosive mixture is in strong chemical disequilibrium but in mechanical equilibrium [5]. The passage of the gas particles through the shock raises the pressure, density and temperature to very high values, so that a chemical reaction starts and proceeds in the reaction zone behind the shock. The following flow, which can be either a constant state (overdriven detonation) or a rarefaction followed by a constant state (unsupported detonation), connects the equilibrium final states at the end of the reaction zone to the rear boundary. All intermediate states of partial reaction are represented by $R$, and the initial state, ahead of the wave, where the mixture is

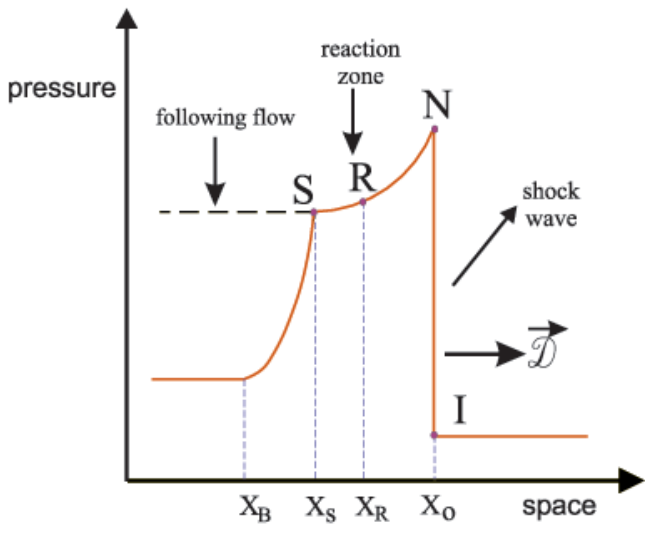

Figure 1: ZND Model for a detonation wave. 
at rest in absolute equilibrium with negligible reaction rate, is denoted by $I$. Moreover $x_{B}$ refers to the rear boundary, $x_{S}, x_{R}$ and $x_{0}$ to the final, intermediate and initial states, respectively. Some modeling aspects of the velocity problem are presented.

Steady governing equations. The RE equations, in the more conservative form of Remark 1, are transformed in the steady frame attached to the shock, moving with velocity $\mathcal{D}$, through the transformation $x^{*}=x-\mathcal{D} t, \tau=t$. Restoring $x$ in place of $x^{*}$, their steady formulation is

$$
\begin{gathered}
(u-\mathcal{D}) \frac{d \varrho_{1}}{d x}+\varrho_{1} \frac{d u}{d x}=m_{1} \widetilde{\tau}_{1}, \\
(u-\mathcal{D}) \frac{d}{d x}\left(n_{1}+n_{3}\right)+\left(n_{1}+n_{3}\right) \frac{d u}{d x}=0, \quad(u-\mathcal{D}) \frac{d}{d x}\left(n_{1}+n_{4}\right)+\left(n_{1}+n_{4}\right) \frac{d u}{d x}=0, \\
(u-\mathcal{D}) \frac{d}{d x}\left(n_{2}+n_{3}\right)+\left(n_{2}+n_{3}\right) \frac{d u}{d x}=0, \quad(u-\mathcal{D}) \frac{d u}{d x}+\frac{1}{\varrho} \frac{d p}{d x}=0, \\
(u-\mathcal{D}) \frac{d}{d x}\left(\frac{3}{2} n k T+\sum_{i=1}^{4} n_{i} E_{i}+\frac{1}{2} \varrho u^{2}\right)+\left(\frac{3}{2} n k T+\sum_{i=1}^{4} n_{i} E_{i}+\frac{1}{2} \varrho u^{2}\right) \frac{d u}{d x}+\frac{d}{d x}(p u)=0 .
\end{gathered}
$$

The Rankine-Hugoniot $(\mathrm{RH})$ conditions, which typically connect the fluxes of independent macroscopic observables preserved across the shock, constitute jump constraints on the state variables $\varrho_{i}, u, p$ between the initial state and an arbitrary state in the reaction zone. Accordingly, the maximal set of steady conservative equations $(18 b-d)$ is integrated between the initial state (subscript 0) and any state in the reaction zone (plain symbols),

$$
\begin{array}{ll}
\left(n_{1}+n_{3}\right)(u-\mathcal{D})=-\left(n_{10}+n_{30}\right) \mathcal{D}, & \left(n_{1}+n_{4}\right)(u-\mathcal{D})=-\left(n_{10}+n_{40}\right) \mathcal{D}, \\
\left(n_{2}+n_{3}\right)(u-\mathcal{D})=-\left(n_{20}+n_{30}\right) \mathcal{D}, & (u-\mathcal{D}) u \sum_{i} m_{i} n_{i}+p=p_{0}, \\
(u-\mathcal{D})\left[u^{2} \sum_{i} m_{i} n_{i}+2 \sum_{i} E_{i} n_{i}+3 p\right]+2 p u=-\left(3 p_{0}+2 \sum_{i} E_{i} n_{i 0}\right) \mathcal{D} .
\end{array}
$$

Steady detonation structure. System (19a-c) will be solved to determine all $N, R$ and $S$ states and the wave thickness, combining an $\mathrm{RH}$ analysis with the rate equation, as described below.

- Von Neumann state $N$. Upstream the shock, since the reaction is not yet initiated, the gas chemical composition does not change across the shock and the progress variable $n_{1}$ is preserved. The rate equation (18a) turns out to be of conservative type, leading to the closure condition

$$
n_{1}(u-\mathcal{D})=-n_{10} \mathcal{D} .
$$

- Intermediate states $R$. Inside the reaction zone the evolution of the state variables depends on the chemical process. The system $(19 a-c)$ is widened with the rate equation (18a) together with its initial condition at the state N, specified by Eq. (20).

- Final state $S$. At the end of the reaction zone where $\tau_{1}=0, S$ is characterized by system $(19 a-c)$ completed by the chemical equilibrium condition, expressed by the mass action law (7). 
- Wave thickness. The length of the reaction zone, which corresponds to the distance between the states $N$ and $S$, can be computed integrating Eq. (18a) from $N$ to $S$ where $\widetilde{\tau}_{1}=0$.

The picture of the detonation wave will be shown in Fig. 2 of Section 6, where the profile of a selected state variable is shown and the wave thickness is plotted in the admissible range of $\mathcal{D}$.

\section{$5 \quad$ Linear stability problem}

The detonation stability is classically studied assuming that a small rear boundary perturbation, instantaneously assigned, induces a distortion on the steady planar shock wave which may either decay with time or grow larger, whereas subsequent rear oscillations do not affect the shock wave [8]. Therefore the shock distortion affects the steady character of the state variables in the reaction zone so that the solution does not admit anymore a steady representation. The response of the steady solution to these perturbations is central for the stability problem which consists of the RE equations in the perturbed shock frame with related RH initial conditions, linearized around the steady wave known from the previous section. The stability solutions provide the behaviour of the state variable disturbances in the reaction zone.

Governing equations in the perturbed shock frame. The choice of such frame is justified since it avoids the risk that hydrodynamic quantities ahead of the wave are exchanged with the corresponding ones behind [9]. Let $\psi(t)$ denote the position of the perturbed shock wave, $\psi(t)=\mathcal{D} t+\psi^{\ell}(t)$, where $\mathcal{D}$ is the constant velocity of the unperturbed shock relative to the laboratory frame, and $\psi^{\ell}(t)$ the distortion on the shock position, so that the perturbed velocity is $\mathcal{D}(t)=\mathcal{D}+\frac{d \psi^{\ell}}{d t}(t)$. In the wave coordinate $x$ which measures the distance from the perturbed front, $x=x^{\ell}-\psi(t)$, the RE equations in the form (14a-c) transform to the system

$$
\begin{aligned}
& \frac{\partial \varrho_{i}}{\partial t}+\left(u-\mathcal{D}-\frac{d \psi^{\ell}}{d t}\right) \frac{\partial \varrho_{i}}{\partial x}+\varrho_{i} \frac{\partial u}{\partial x}=-m_{i} \nu_{i} \widetilde{\tau}_{1}, \quad i=1, \ldots, 4, \\
& \frac{\partial u}{\partial t}+\left(u-\mathcal{D}-\frac{d \psi^{\ell}}{d t}\right) \frac{\partial u}{\partial x}+\frac{1}{\varrho} \frac{\partial p}{\partial x}=0, \quad \frac{\partial p}{\partial t}+\left(u-\mathcal{D}-\frac{d \psi^{\ell}}{d t}\right) \frac{\partial p}{\partial x}+\frac{5}{3} p \frac{\partial u}{\partial x}=\eta,
\end{aligned}
$$

or to a matrix form which will be more useful in the sequel

$$
\frac{\partial \boldsymbol{z}}{\partial t}+\left[A(\boldsymbol{z})-\left(\mathcal{D} I+\frac{d \psi^{\ell}}{d t}\right) I\right] \frac{\partial \boldsymbol{z}}{\partial x}=\boldsymbol{c}(\boldsymbol{z})
$$

where $\boldsymbol{z}, A, \boldsymbol{c}$ are the same as in Eqs. (15) and (16). The RH conditions (19a-c) and (20), re-written in terms of the state variables and in the wave coordinate, transform to

$$
\begin{aligned}
& \left(u-\mathcal{D}-\frac{d \psi^{\ell}}{d t}\right) \varrho_{i}=-\left(\mathcal{D}+\frac{d \psi^{\ell}}{d t}\right) \varrho_{i 0}, \quad\left(u-\mathcal{D}-\frac{d \psi^{\ell}}{d t}\right) u \sum_{i} \varrho_{i}+p=p_{0}, \\
& \left(u-\mathcal{D}-\frac{d \psi^{\ell}}{d t}\right)\left[u^{2} \sum_{i} \varrho_{i}+2 \sum_{i} \frac{E_{i}}{m_{i}} \varrho_{i}+3 p\right]+2 p u-\left(3 p_{0}+2 \sum_{i} \frac{E_{i}}{m_{i}} \varrho_{i 0}\right)\left(\mathcal{D}+\frac{d \psi^{\ell}}{d t}\right) .
\end{aligned}
$$

Equation (22) and initial conditions (23a-b) constitute the governing equations of the model. 
Normal mode analysis. The governing equations are linearized assuming that the shock distortion and state variables perturbation have an exponential time dependence [3]. A normal mode expansion about the steady solution approximates the state vector, the shock distortion and their derivative as follows

$$
\begin{array}{clrl}
\boldsymbol{z}(x, t) & =\boldsymbol{z}^{*}(x)+\exp (a t) \overline{\boldsymbol{z}}(x), \quad \psi^{\ell}(t)=\bar{\psi} \exp (a t), & a, \bar{\psi} \in \mathbb{C}, & a=\alpha+i \beta, \\
\frac{\partial \boldsymbol{z}}{\partial t} & =a \exp (a t) \overline{\boldsymbol{z}}, \quad \frac{\partial \boldsymbol{z}}{\partial x}=\frac{d \boldsymbol{z}^{*}}{d x}+\exp (a t) \frac{d \overline{\boldsymbol{z}}}{d x}, & \frac{d \psi^{\ell}}{d t} & =a \bar{\psi} \exp (a t),
\end{array}
$$

where $\overline{\boldsymbol{z}}=\left[\bar{\varrho}_{1} \bar{\varrho}_{2} \bar{\varrho}_{3} \bar{\varrho}_{4} \bar{u} \bar{p}\right]^{\mathrm{T}}$, the bar means unknown disturbances, the star denotes the known steady state, $\operatorname{Re} a$ and $\operatorname{Im} a$ represent the perturbation growth rate and frequency. The sign of Re $a$ determines the stability behaviour of the steady solution.

Linearized RE equations in the wave coordinate. Inserting expansions (24) and (25) in Eqs. $(21 a-b)$ the linearized equations for the perturbations $\bar{z}$ are written as

$$
\left(A^{*}-\mathcal{D} I\right) \frac{d \overline{\boldsymbol{z}}}{d x}+\left(a I+C^{*}\right) \overline{\boldsymbol{z}}-a \boldsymbol{b}^{*} \bar{\psi}=0,
$$

where $A^{*}=A\left(\boldsymbol{z}^{*}\right), \boldsymbol{b}^{*}=\frac{d z^{*}}{d x}=\left[\begin{array}{llllll}\frac{d \varrho_{1}^{*}}{d x} & \frac{d \varrho_{2}^{*}}{d x} & \frac{d \varrho_{3}^{*}}{d x} & \frac{d \varrho_{4}^{*}}{d x} & \frac{d u^{*}}{d x} & \frac{d p^{*}}{d x}\end{array}\right]^{\mathrm{T}}$, and $C^{*}$ is a matrix defined in terms of $\boldsymbol{z}^{*}$, whose non-zero elements are

$$
\begin{gathered}
C_{i j}^{*}=\delta_{i j} \frac{d u^{*}}{d x}-\nu_{i} m_{i} \widehat{S}^{*} \varrho_{h}^{*} \Theta, j=1,2, \quad C_{i j}^{*}=\delta_{i j} \frac{d u^{*}}{d x}+\nu_{i} m_{i} \widehat{S}^{*} \varrho_{h}^{*}, j=3,4, \quad i=1, \ldots, 4, \\
C_{5 j}^{*}=\frac{u^{*}-\mathcal{D}}{\varrho^{*}} \frac{d u^{*}}{d x}, j=1, \ldots, 4, \quad C_{i 5}^{*}=\frac{d z_{i}^{*}}{d x}, i=1, \ldots, 6, \\
C_{6 j}^{*}=\frac{2 E}{3} \widehat{S}^{*} \varrho_{h}^{*} \Theta, j=1,2, \quad C_{6 j}^{*}=-\frac{2 E}{3} \widehat{S}^{*} \varrho_{h}^{*}, j=3,4, \quad C_{66}^{*}=\frac{5}{3} \frac{d u^{*}}{d x} .
\end{gathered}
$$

Above, $\delta_{i j}$ is the Kronecker symbol, the index $h$ is such that $h=2,1,4,3$ when $j=1,2,3,4$, respectively, and $\Theta=\left(\frac{m_{3} m_{4}}{m_{1} m_{2}}\right)^{5 / 2} \exp \left(-\frac{n^{*} E}{p^{*}}\right)$.

Linearized RH initial conditions in the wave coordinate. The initial conditions to be joined to the differential Eqs. (26) relate the value of the disturbances $\bar{z}_{\mathrm{N}}$ behind the shock to their zero value at the initial state ahead. The linearization of the RH conditions (19a-b) and (20) trough expansions (24) and (25) 3 lead to

$$
\left(A_{\mathrm{N}}^{*}-\mathcal{D} I\right) \overline{\boldsymbol{z}}_{\mathrm{N}}=a \bar{\psi}\left(K_{\mathrm{N}}^{*} \boldsymbol{z}_{\mathrm{N}}^{*}-\boldsymbol{z}_{0}\right),
$$

where $\boldsymbol{z}_{0}=\left[\begin{array}{llllll}\varrho_{10} & \varrho_{20} & \varrho_{30} & \varrho_{40} & 0 & p_{0}\end{array}\right]^{T}$ is the state vector in the initial state $I$ and the non-zero elements of $K_{\mathrm{N}}^{*}$ are

$$
K_{i i}^{*}=1, \quad i=1,2,3,4,6, \quad K_{55}^{*}=\sum_{i} \varrho_{i 0} / \sum_{i} \varrho_{i}^{*}, \quad K_{65}^{*}=-\sum_{i} \varrho_{i 0} u_{N}^{*} / 3 .
$$


Stability problem and its determinacy. Equations (26) and (27) can be re-written avoiding the dependence of $\overline{\boldsymbol{z}}$ and $\overline{\boldsymbol{z}}_{\mathrm{N}}$ on the complex parameter $\bar{\psi}$ through the normalization $\overline{\boldsymbol{\zeta}}=\overline{\boldsymbol{z}} / \bar{\psi}$, as

$$
\begin{gathered}
\left(A^{*}-\mathcal{D} I\right) \frac{d \overline{\boldsymbol{\zeta}}}{d x}+\left(a I+C^{*}\right) \overline{\boldsymbol{\zeta}}-a \boldsymbol{b}^{*}=0, \quad x \in\left[x_{\mathrm{N}}, x_{\mathrm{eq}}\right], \\
\overline{\boldsymbol{\zeta}}_{\mathrm{N}}=a\left(A_{\mathrm{N}}^{*}-\mathcal{D} I\right)^{-1}\left(K_{\mathrm{N}}^{*} \boldsymbol{z}_{\mathrm{N}}^{*}-\boldsymbol{z}_{0}\right) .
\end{gathered}
$$

They constitute the stability system for the detonation wave in 12 unknowns $\operatorname{Re} \overline{\boldsymbol{\zeta}}$ and $\operatorname{Im} \overline{\boldsymbol{\zeta}}$, which in general is not closed since the complex parameter $a$ is a priori unknown. In paper [1] a closure condition has been rigorously derived for two-dimensional perturbations, and has been proven to be equivalent to the physical requirement of the acoustics radiation condition for inert flows, originally derived in paper [10]. This constraint, traditionally used in detonation literature, expresses the physical condition for wich no waves emanate from the boundary to interfere with the steady character of the lead shock, so that the reaction zone is acoustically isolated from the following flow. Accordingly, observing that at the equilibrium the gas behaves like an inert flow, the closure condition is obtained as

$$
\bar{\zeta}_{5}+a=\frac{-1}{\gamma \varrho_{\mathrm{eq}}^{*} c_{\mathrm{eq}}^{*}} \bar{\zeta}_{6}, \quad \text { for } \quad x=x_{\mathrm{eq}} .
$$

where $c_{\mathrm{eq}}^{*}$ is the isentropic sound speed and $\varrho_{\mathrm{eq}}^{*}$ the gas density at equilibrium, and $\gamma$ the ratio of specific heats.

Solution technique. The stability problem (28), (29) and (30) is now treated numerically to compute complex eigenvalues $a$ and eigenfunctions $\bar{\zeta}$, applying a technique detailed as follows.

- For several values of $a$ in a bounded domain of the right complex half-plane, Eqs. (28) are integrated with initial conditions (29), using a fourth order Runge-Kutta routine.

- The solution $\overline{\boldsymbol{\zeta}}(x), x \in\left[x_{\mathrm{N}}, x_{\mathrm{eq}}\right]$, obtained for trial values of $a$ is specialized for $x=x_{\mathrm{eq}}$ to estimate the residual function $|H(a)|$, with $H(a)$ defined from the radiation condition (30) as

$$
H(a)=\bar{\zeta}_{5}+a+\frac{1}{\gamma \varrho_{\mathrm{eq}}^{*} c_{\mathrm{eq}}^{*}} \bar{\zeta}_{6} .
$$

- Only those solutions $\overline{\boldsymbol{\zeta}}$, for which $|H(a)|$ results to be close to zero within an acceptable tolerance, are taken into account for the stability analysis.

The eigenfunctions selected according to the last step allow to evaluate the influence of the perturbations induced by the shock distortion on the steady solution $\boldsymbol{z}^{*}$. It is enough to refer the decomposition $(24)_{1}$ to the stability solutions $\overline{\boldsymbol{\zeta}}$, namely

$$
\boldsymbol{z}(x, t)=\boldsymbol{z}^{*}(x)+\exp (a t) \overline{\boldsymbol{\zeta}}(x) .
$$

If now $\boldsymbol{\sigma}(x)=\operatorname{Re}, \overline{\boldsymbol{\zeta}}(x), \boldsymbol{\tau}(x)=\operatorname{Im} \overline{\boldsymbol{\zeta}}(x), \boldsymbol{\Gamma}=\exp (a t) \overline{\boldsymbol{\zeta}}(x)$, the influence of the disturbances $\overline{\boldsymbol{\zeta}}$ on $z^{*}$ is described by the behaviour of $\operatorname{Re} \Gamma_{i}, \operatorname{Im} \Gamma_{i}, i=1, \ldots, 6$. Therefore the real and imaginary parts of the disturbances of the total mass density, pressure and mean velocity are provided by

$$
\sum_{i=1}^{4} \operatorname{Re} \Gamma_{i}=e^{\alpha t} \sum_{i=1}^{4}\left(\sigma_{i}(x) \cos \beta t-\tau_{i}(x) \sin \beta t\right), \quad \sum_{i=1}^{4} \operatorname{Im} \Gamma_{i}=e^{\alpha t} \sum_{i=1}^{4}\left(\sigma_{i}(x) \sin \beta t+\tau_{i}(x) \cos \beta t\right),
$$




$$
\begin{gathered}
\operatorname{Re} \Gamma_{5}=e^{\alpha t}\left(\sigma_{5}(x) \cos \beta t-\tau_{5}(x) \sin \beta t\right), \quad \operatorname{Re} \Gamma_{6}=e^{\alpha t}\left(\sigma_{6}(x) \cos \beta t-\tau_{6}(x) \sin \beta t\right), \\
\operatorname{Im} \Gamma_{5}=e^{\alpha t}\left(\sigma_{5}(x) \sin \beta t+\tau_{5}(x) \cos \beta t\right), \quad \operatorname{Im} \Gamma_{6}=e^{\alpha t}\left(\sigma_{6}(x) \sin \beta t+\tau_{6}(x) \cos \beta t\right) .
\end{gathered}
$$

In the next section, some results concerning the behaviour of the real disturbances are provided within a picture of either stable or unstable modes. Conversely, the behaviour of the imaginary disturbances is inspected for the neutral stability boundaries corresponding to $\alpha=0$.

\section{Results and discussion}

In this section, some simulations relative to both steady detonation wave and its linear stability are developed for the elementary reaction of the Hydrogen-Oxygen chain, $\mathrm{H}_{2} \mathrm{O}+\mathrm{H} \rightleftharpoons \mathrm{OH}$ $+\mathrm{H}_{2}$. The initial state of the fresh unreacted mixture with number densities $n_{i}$ (in $m o l l^{-1}$ ), temperature $T$ (in $K$ ), and the reference data for the masses $m_{i}$ (in $\mathrm{kg} \mathrm{mol}$ ), scale factor $\theta$ of the chemical collision frequency, threshold velocity $\chi$ (in $\mathrm{ms}^{-1}$ ) of interacting particles and binding energy difference $E$ (in $J m_{o l}{ }^{-1}$ ), are given by are

$$
\begin{gathered}
n_{1}=0.03, n_{2}=0.02, n_{3}=0.10, n_{4}=0.20, T=298.15, \\
m_{1}=0.018, m_{2}=0.001, m_{3}=0.017, m_{4}=0.002, \theta=10^{7}, \chi=6851, E=63311 .
\end{gathered}
$$

Results on steady detonation. Applying the procedure outlined in Section 4, the velocity problem has been solved on the basis of the description carried out in paper [5], for a detonation velocity $\mathcal{D}=3500$, greater than the Chapman-Jouguet velocity $\mathcal{D}_{J}=2973,5$, that is for an overdrive degre $f=\mathcal{D} / \mathcal{D}_{J}=1.3854$. The wave structure is represented by its thickness curve and number density profile. In Fig.2 (left), the decaying behaviour of the wave thickness, $z_{\mathrm{F}}$, is shown versus $f$. In Fig.2 (right), the total number density is represented versus the wave propagation coordinate.

Results on linear stability. The analysis of Section 5 is applied to the steady solution above described. For a growth rate $\alpha=0.01$ and disturbance frequency $\beta=0.01$, Fig. 3 and Fig. 4 (left) show the amplification in time of the unstable modes of the real parts of the disturbances, namely $\sum_{i=1}^{4} \operatorname{Re} \Gamma_{i}, \operatorname{Re} \Gamma_{5}$ and $\operatorname{Re} \Gamma_{6}$ for the von Neuman state $(V N)$, an intermediate state $(I)$ and the equilibrium final state $(F)$. For sake of simplicity and having in mind their physical meaning in terms of the macroscopic observables, such disturbances are labelled in the figures by $\bar{\varrho}, \bar{u}$ and $\bar{p}$, respectively. The pictures reveal that the amplitudes of $\bar{\rho}$ are greater at the von Neuman state and smaller at the final state, the opposite occurs for $\bar{p}$ and $\bar{u}$. The unstable picture is completed by Fig.4 (right) where a further significant feature is put in evidence, confirming the existence of perturbation modes more unstable for decreasing values of frequency $\beta$, at fixed positive growth rate $\alpha=0.01$. This behaviour is in agreement with some relevant results shown in paper [3].

Conversely, a stability picture is exhibited in Fig.5 (left) where a rapid decay in time of the perturbation amplitude of $\bar{\varrho}$, for different states within the reaction zone, is drawn for $\alpha=-0.01$ and $\beta=0.01$. All other profiles rapidly converge to zero, showing a quite similar trend.

At conditions of neutral stability $(\alpha=0)$, Fig.5 (right) shows the trend to constant values of the perturbations $\sum_{i=1}^{4} \operatorname{Im} \Gamma_{i}, \operatorname{Im} \Gamma_{5}$ and $\operatorname{Im} \Gamma_{6}$, which are labelled with $\operatorname{Im} \xi, \operatorname{Im} \xi_{5}$ and $\operatorname{Im} \xi_{6}$. 

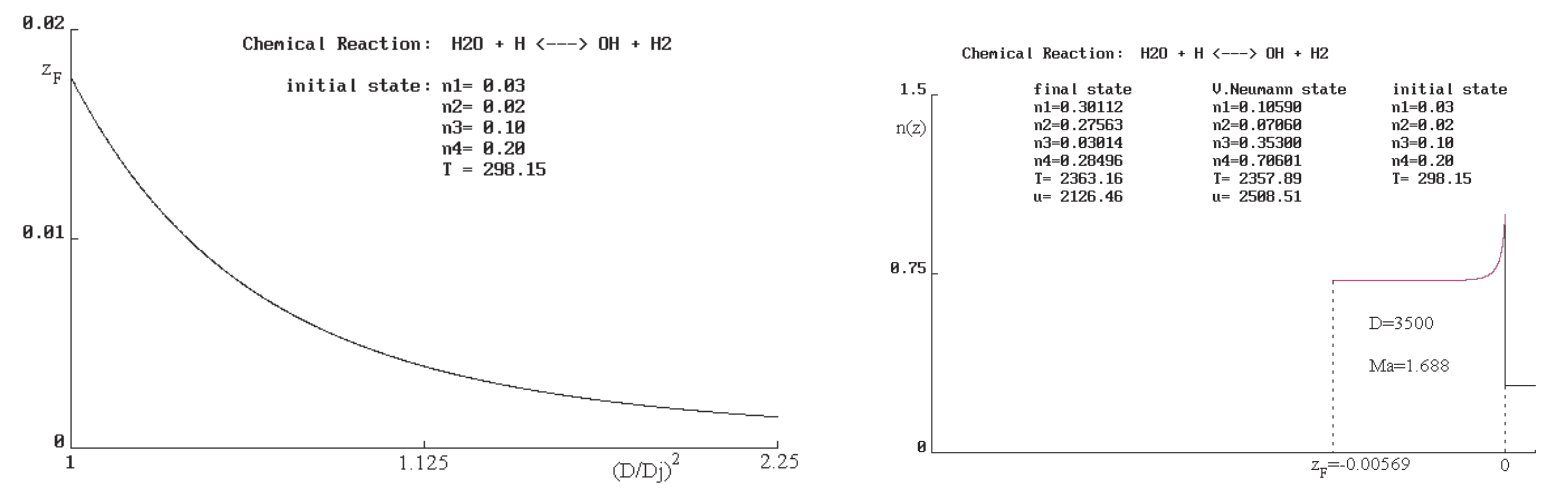

Figure 2: Steady detonation solution - Decay of the wave thickness $z_{F}$ versus overdrive degree (left) and profile of total number density $n$ for $f=1.3854$ (right).

Conclusions. The results obtained here with the above described simulations are in qualitative agreement with those known in detonation literature concerning the one dimensional linear stability, even though they should be considered a first attempt in kinetic theory which can be extended to the case of bi-dimensional disturbances induced by a shock distortion of type $\psi^{\ell}(y, t)$. Thus it seems promising to develop a more complete hydrodynamic stability analysis in order to go deeper on the effects due to the heat release and activation energy of the chemical reaction. Within kinetic modelling of reacting flows, the present study shows that it is possible to deduce a correct mathematical formulation of the complex problem of the detonation stability. At the same time, this work contributes to extend the spectrum of fluid hydrodynamic applications, based on Boltzmann type equations, to the most relevant non equilibrium effects induced by chemical reactions.

\section{Acknowledgements}

The authors are grateful to Prof. R. Monaco for his precious help in the numerical simulations. The paper is partially supported by INDAM-GNFM and CMAT-FCT Minho University.

\section{References}

[1] A. Kasimov and D. S. Stewart, "Spinning instability of gaseous detonations", J. Fluid Mech., 466, 179-203 (2002).

[2] F. Conforto, R. Monaco, F. Schürrer and I. Ziegler, "Steady detonation waves via the Boltzmann equation for a reacting mixture", J. Phys. A: Math. Gen., 36, 5381-5398 (2003).

[3] M. Short and D. S. Stewart, "Multidimensional linear stability of a detonation of a detonation wave at high activation energy", SIAM J. Appl. Math., 57, 307-326 (1997). 

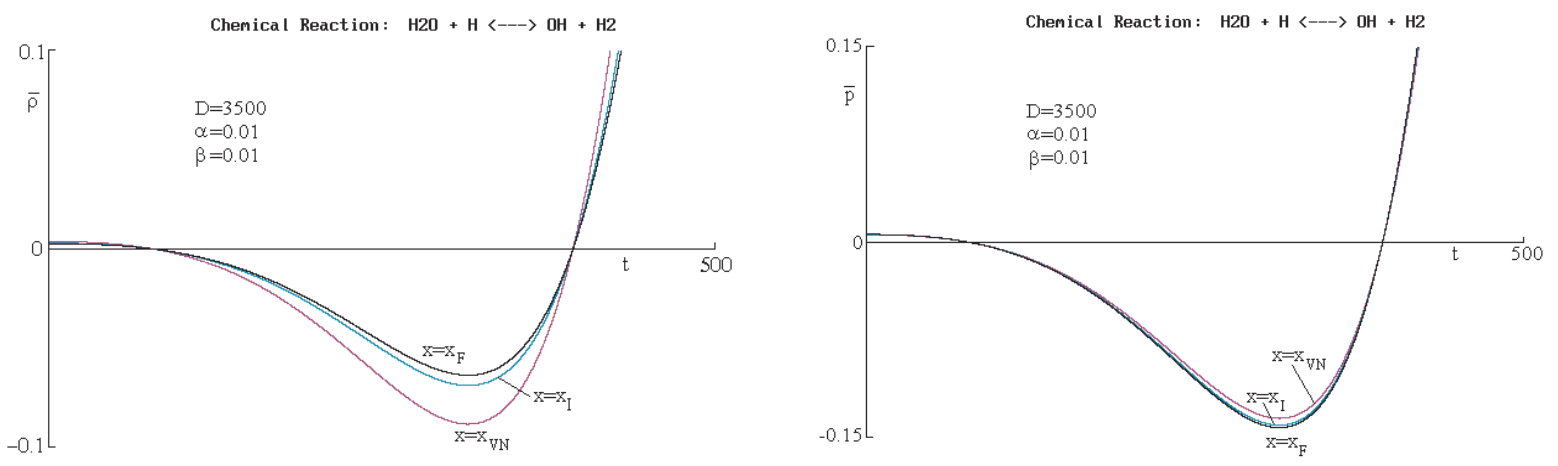

Figure 3: Instability behaviour for growth rate Re $a=0.01$. Time evolution of the real parts of the disturbance amplitudes $\bar{\rho}$ (left) and $\bar{p}$ (right), at von Neuman $\left(x=x_{\mathrm{N}}\right)$, intermediate $\left(x=x_{\mathrm{I}}\right)$ and equilibrium final state $\left(x=x_{\mathrm{F}}\right)$.
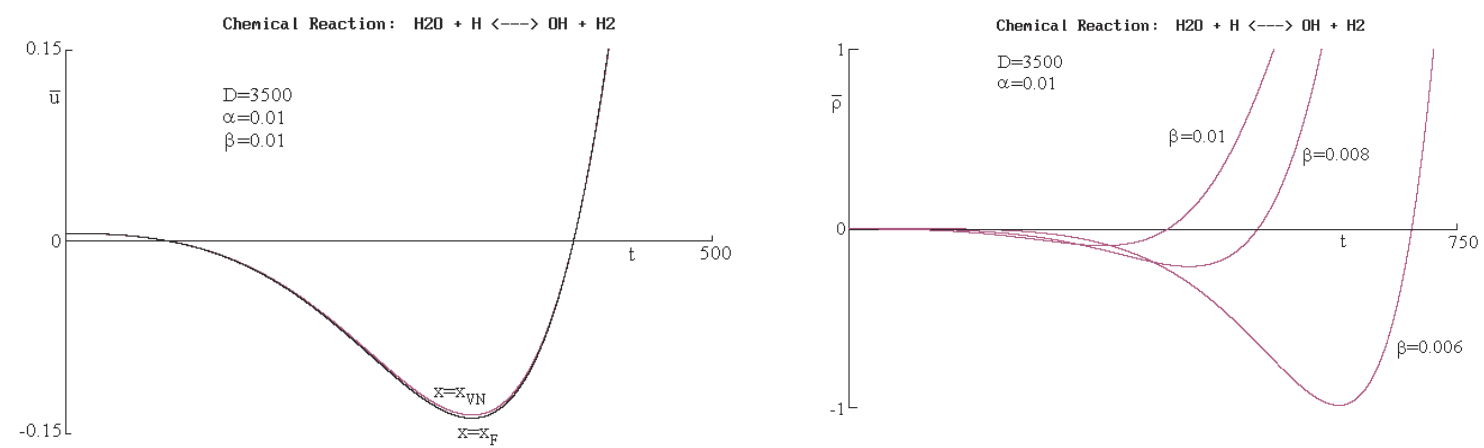

Figure 4: Instability behaviour for $\operatorname{Re} a=0.01$. Left - Time evolution of the real parts of the disturbance amplitudes $\bar{u}$ at von Neuman $\left(x=x_{\mathrm{N}}\right)$ and final state $\left(x=x_{\mathrm{F}}\right)$. Right-Time evolution of the real part of the disturbance amplitude $\bar{\rho}$ for decreasing perturbation frequencies $\beta$. 

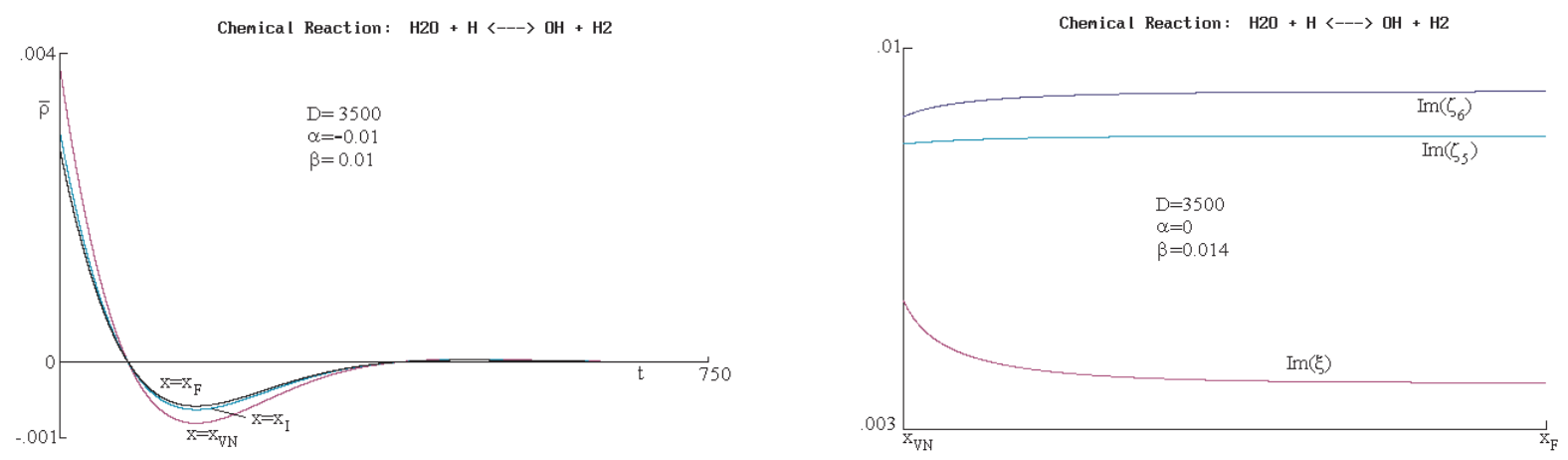

Figure 5: Stability behaviour for Re $a=-0.01$ - time decay of the real parts of the disturbance amplitudes for $\bar{\rho}$ (left) at von Neuman $\left(x=x_{\mathrm{N}}\right)$, intermediate $\left(x=x_{\mathrm{I}}\right)$ and equilibrium final state $\left(x=x_{\mathrm{F}}\right)$. Neutral stability $(\operatorname{Re} a=0)$ profiles of imaginary parts of the disturbance amplitudes (right).

[4] M. Bisi, M. Groppi, G. Spiga, "A kinetic model for bimolecular reactions", in Kinetic Methods for Non Conservative and Reacting Systems, Ed. G. Toscani, Aracne Ed. Roma 16, 1-145 (2005).

[5] F. Conforto, M. Groppi, R. Monaco, G. Spiga, "Steady detonation waves for gases undergoing dissociation/recombination and bimolecular reactions", Continuum Mech. Thermodyn, 16, 149-161 (2004).

[6] I. Prigogine and R. Defay, Chemical Thermodynamics, Longman, London (1973).

[7] P. Griehsnig, F. Schürrer, G. Kügerl, "Kinetic theory for particles with internal degrees if freedom" Rarefied Gas Dynamics, Eds. B. D. Shizgal, D. P. Weaver, AIAA, 159, 581-589 (1992).

[8] A. Kasimov and D. S. Stewart, "Theory of detonation with an embedded sonic locus", SIAM J. Appl. Math., 66, 384-407 (2005).

[9] W. Fickett, Introduction to Detonation Theory, University of California, Berkeley (1986).

[10] J. D. Buckmaster and G. S. S. Ludford, "The effect of structure on the stability of detonations I. role of the induction zone", Proc. XX Simp. Comb., 1669-1676 (1986). 